\title{
APPLICATION OF NEUTRON REFRACTION FOR CHARACTERIZATION AND OPTIMIZATION OF HIGH-DAMPING IRON-BASED ALLOYS
}

\author{
V.A. Udovenko ${ }^{a}$, N.A. Polyakova $^{a}$, I.B. Chudakov ${ }^{a}$, J.J. MilczareK ${ }^{b}$ \\ AND K. MIKKE ${ }^{b}$ \\ ${ }^{a}$ I.P. Bardin State Scientific Centre, Moscow 107005, Russia \\ ${ }^{b}$ Institute of Atomic Energy, 05-400 Otwock-Świerk, Poland
}

\begin{abstract}
High-damping alloys with the magnetomechanical damping mechanism, based on the $\mathrm{Fe}-\mathrm{Cr}$ system, have been studied. It has been shown that the direct investigation of the magnetic domain structure in the bulk materials using the neutron refraction method allows one to obtain important quantitative information concerning the formation of the high-damping state. The dependence of the damping capacity on the average magnetic domain size was found to possess a sharp maximum, thus revealing a range of the optimal domain sizes. The results obtained are important for the development of the technology of high-damping materials.
\end{abstract}

PACS numbers: $61.12 .-\mathrm{q}, 75.50 . \mathrm{Bb}, 75.60 . \mathrm{Ej}, 75.80 .+\mathrm{q}$

\section{Introduction}

Some of the Fe-based alloys are known to exhibit very high-damping capacity in the range of small vibration amplitudes due to the stress-driven irreversible movement of the $90^{\circ}$-magnetic domain walls (DWs) $[1,2]$. It is noteworthy that the level of damping capacity $\psi$ of the materials with the magnetomechanical damping mechanism may exceed $50 \%$, where a half of the total vibration energy is dissipated per one cycle of oscillation $[3,4]$. This is one of the reasons, why this class of materials is considered to be a very interesting object for the investigation.

Unfortunately, little information is available in the literature concerning the magnetic domain structure (MDS) of the alloys with a high-damping capacity, but the main attention has focused on the local interaction of $90^{\circ}$-DWs with different kinds of obstacles to the motion of individual DW [1-4].

It must be specially mentioned that MDS of high-damping Fe-based alloys has not been studied carefully in the bulk materials. This should be done especially for the materials with the three easy magnetization axes, where the surface MDS-patterns do not provide sufficient information concerning the domain 
structure in the bulk due to the appearance of the closure and supplementary domains $[5,6]$.

The studies of the special features of MDS in the bulk samples of high-damping $\mathrm{Fe}-\mathrm{Cr}$ alloys have been the aim of the present work.

\section{Experimental}

The alloys of the $\mathrm{Fe}-\mathrm{Cr}$ system were selected for the investigation as the most representative ferritic Fe-based alloys with magnetomechanical damping of a high level. These alloys have been widely investigated previously with the help of a set of modern experimental techniques $[1-4,7,8]$.

Materials have been prepared using a standard metallurgical technology, including vacuum-melting from high-purity raw materials, forging and rolling [8]. Let us mention that the level of impurities and nonmetallic inclusions did not exceed the following limits: $0.009 \% \mathrm{C}, 0.13 \% \mathrm{Si}, 0.05 \% \mathrm{Mn}, 0.01 \% \mathrm{Ti}$ (wt.\%).

The neutron refraction investigation has been performed using the double-crystal small angle neutron scattering spectrometer with a high angular resolution. The neutron wavelength $\lambda$ was equal to $0.155 \mathrm{~nm}$. The inverted bending pendulum method was used to study the damping capacity.

\section{Experimental results and discussion}

Figure 1 presents typical amplitude dependences of damping capacity of the $\mathrm{Fe}-\mathrm{Cr}$ alloys. It can be seen that the value of the logarithmic decrement $\delta$ can be remarkably changed ( $\sim 10$ times) using the variation of the heat treatment regime. The drastic change of the damping capacity was found to be caused by essential modifications in the crystalline $[2-4,8]$ and magnetic $[7,8]$ structure of the material. In the present paper, let us briefly present the generalized data

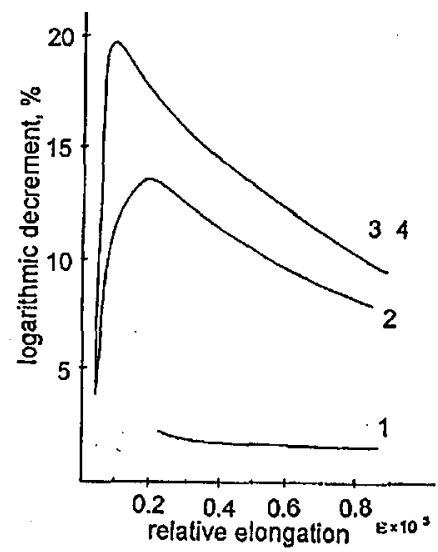

Fig. 1. Amplitude dependences of damping capacity for the alloy $\mathrm{Fe}-16 \mathrm{Cr}-0.02 \mathrm{Ti}$. Heat treatment: 1 - water quenching from $T=10000^{\circ} \mathrm{C} ; 2-$ air-cooling; 3 - furnace cooling; 4 - cooling from $T=1000$ to $600^{\circ} \mathrm{C}$ with the furnace, then cooling on the air. 
concerning the interrelation between the damping capacity and the average size of magnetic domains. It is noteworthy that the neutron refraction method allows one to obtain ensemble-averaged characteristics of MDS of the bulk polycrystalline samples (dimensions up to $6 \mathrm{~mm}$ in the case of $\mathrm{Fe}-\mathrm{Cr}$ alloys).

Figure 2 presents the histogram that displays the dependence of the maximum value of the logarithmic decrement on the average domain size in the bulk samples. These two parameters are seen to correlate quite well. A distinct maximum, close to $h_{\mathrm{d}} \approx 8 \mu \mathrm{m}$, can be observed as well as the optimal $h_{\mathrm{d}}$ range (from 7 to $9 \mu \mathrm{m}$ ) for the realization of the high-damping state. It is important that the histogram in Fig. 2 represents data obtained for several alloys on the base of the $\mathrm{Fe}-\mathrm{Cr}$ system, both binary $(\sim 15-18 \% \mathrm{Cr})$ and alloyed with $\mathrm{Mo}, \mathrm{Nb}$, and $\mathrm{Ni}$, after various heat treatment regimes. Therefore, it can be assumed that this regularity is quite general for the $\mathrm{Fe}-\mathrm{Cr}$ alloys, and is one of the important factors in the formation of the high-damping state.

A detailed investigation of the heat treatment effect on the average domain size has been carried out using the $\mathrm{Fe}-16 \mathrm{Cr}-0.2 \mathrm{Nb}$ alloy. It has been found that the decrease in the cooling rate during the heat treatment causes a decrease in $h_{\mathrm{d}}$.

Taking into account that this tendency was found to be valid for all alloys tested, the following assumption can be made. A decrease in the cooling rate, apparently, is equivalent to the motion from right to left along the $x$-axis of the histogram, presented in Fig. 2. In such a way, if we select the cooling rate during the heat treatment so as to obtain the optimal $h_{\mathrm{d}}$ value (about $8 \mu \mathrm{m}$ ), then the high level of damping capacity shall be realized in the investigated alloys $\left(\delta_{\max } \approx\right.$ $15-20 \%$ or higher). This special feature allows effective optimization of the heat treatment regimes for model and industrial high-damping alloys, based on the $\mathrm{Fe}-\mathrm{Cr}$ system.

It should be noted that not only the damping capacity, but also the coercive force of material was found to exhibit the correlation with the average domain size, obtained using the neutron refraction method. A histogram illustrating the dependence of the coercive force on $h_{\mathrm{d}}$ is presented in Fig. 3. It can be seen that $H_{\mathrm{c}}$ decreases with an increase in $h_{\mathrm{d}}$. This dependence may be explained by the

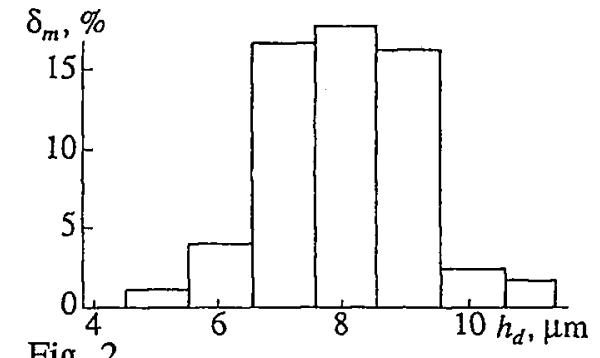

Fig. 2

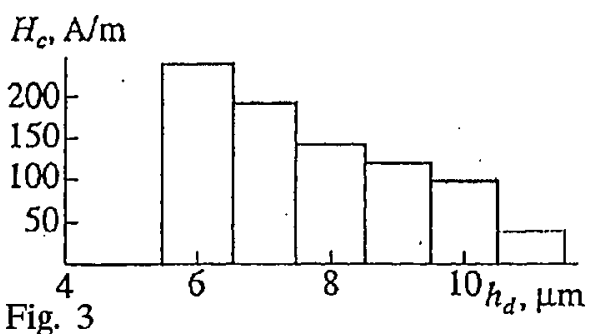

Fig. 3

Fig. 2. Histogram, illustrating dependence of maximum logarithmic decrement on the average magnetic domain size.

Fig. 3. Histogram, illustraing dependence of the coercive force on the a verage magnetic domain size. 
increase in domain walls density with the decrease in $h_{\mathrm{d}}$ (taking into account the additivity of local losses, produced by the movement of individual domain wall). A comparison of Figs. 2 and 3 allows us to match the range of optimum domain size with the range of optimum coercive force $\left(H_{\mathrm{c}}=120 \div 240 \mathrm{~A} / \mathrm{m}\right)$.

In such a manner three material parameters were found to be in a close connection: the damping capacity, the coercive force, and the average size of magnetic domains. This result, obviously, is very important to understand the processes, responsible for the formation of the high-damping state in ferromagnetic materials with the three easy magnetization axes.

\section{Conclusions}

1. Application of the neutron refraction method for the studies of high-damping Fe-based alloys allows one to obtain important quantitative information concerning the formation of the high-damping state as well as concerning special features of the magnetic domain structure.

2. The correlation between the damping capacity and the average size of magnetic domains in the bulk samples has been established. The range of average domain size, optimal for the formation of high-damping state in the $\mathrm{Fe}-\mathrm{Cr}$ alloys, was found to be relatively narrow, namely from 7 to $9 \mu \mathrm{m}$.

3. Neutron refraction data give a good basis for the optimization of the heat treatment regimes of model and industrial high-damping $\mathrm{Fe}-\mathrm{Cr}$ alloys.

4. The decrease in the average size of magnetic domains causes the increase in the coercive force of high-damping alloys on the base of the $\mathrm{Fe}-\mathrm{Cr}$ system.

\section{References}

[1] A. Cochardt, in: Magnetic Properties of Metals and Alloys, American Soc. Metals, Cleveland (Ohio) 1959, p. 328.

[2] I.B. Kekalo, Phys. Met. Heat Treat. 7, 5 (1973).

[3] Yu.K. Favstov, Phys. Met. Heat Treat. 18, 98 (1984).

[4] J. Degauque, unpublished.

[5] S. Chikasumi, Physics of Ferromagnetism. Magnetic Characteristics and Engineering Applications, Syokabo, Tokyo 1984, p. 287.

[6] R.M. Bozorth, Ferromagnetism, D. Van Nostrand, New York 1951, p. 420.

[7] V.A. Udovenko, Nukleonika 39, 149 (1994).

[8] V.A. Udovenko, I.B. Chudakov, N.A. Polyakova, Phys. Met. Metallogr. 75, 247 (1993). 